\title{
Artes y humanidades en la formación de personal de salud y en la atención integral en un hospital universitario
}

\author{
| ${ }^{1}$ Ana María Medina Ch., ${ }^{2}$ María Buitrago, ${ }^{3}$ Liliana Ramirez , ${ }^{4}$ Jennifer Clavijo |
}

Resumen: El objetivo de este artículo es presentar las múltiples formas de aplicación de las artes y las humanidades en contextos hospitalarios y en la formación profesional en las Facultades de Medicina y Enfermería y un Hospital Universitario de la ciudad de BogotáColombia en el periodo 2008-2018. La caracterización se hizo a partir de métodos cualitativos, interpretativos, con aplicación de entrevistas semiestructuradas a actores identificados por bola de nieve y auto reporte. También se hizo observación participante en algunos lugares y prácticas. Se indagó sobre los sentidos y usos de las artes y humanidades con relación a la salud y la formación en salud. Los diversos actores asignan sentidos a estas prácticas relacionándolas con una mayor receptividad en el proceso enseñanza aprendizaje, la posibilidad de generar sentido de trascendencia sobre lo que se hace, dar respuesta a preguntas existenciales, proporcionar bienestar a las personas en condiciones de hospitalización y en menor medida a generar bienestar propio o de los profesionales de salud. Su lugar en los contextos explorados aún se percibe como subalterno, y se sostienen en su mayoría a partir de iniciativas individuales y voluntariado. Debe insistirse en su legitimidad para avanzar en su sentido social y emancipador.

Palabras-clave: Humanidades, Artes, Salud, Educación médica, voluntarios.

\author{
1 Pontificia Universidad Javeriana. \\ Bogotá - Colombia (medina.ana@ \\ javeriana.edu.co). \\ ORCID: 0000-0002-9132-0557 \\ ${ }^{2}$ Fundacion Universitaria del \\ Area Andina. Bogotá - Colombia \\ (Mbuitrago36@areandina.edu.co). \\ ORCID: 0000-0002-6667-5874 \\ 3 Pontificia Universidad Javeriana. \\ Bogotá - Colombia (liliana- \\ ramirez@javeriana.edu.co). \\ ORCID: 0000-0002-9270-9214 \\ 4 Pontificia Universidad Javeriana. \\ Bogotá - Colombia (jennifer. \\ clavijo@javeriana.edu.co). \\ ORCID: 0000-0003-1264-3778
}

Recebido em: 18/08/2020 Aprovado em: 22/10/2020 Revisado em: 08/10/2021 


\section{Introducción}

A nivel mundial las humanidades médicas en contextos hospitalarios han abierto un espacio de investigación que por excelencia es interdisciplinar. Si bien no existe una definición estática o categorización que permita identificar qué hace parte y qué no de las humanidades médicas, se entiende de manera generalizada que es un campo interdisciplinario que abarca las diversas artes y formas expresivas, así como algunas ciencias sociales y humanas en particular la literatura (PINTO; JORGE, 2013), en relación a la atención en salud. Se dice que las humanidades brindan marcos críticos, reflexivos, complejos y profundos sobre la realidad vivida (BLEASE, 2016). Otras competencias como la introspección, reflexión y aprendizaje de capacidades críticas parecen desarrollarse a través de las humanidades y las artes en la formación del personal de salud (BARBER; MORENO-LEGUIZAMON, 2017; BLEASE, 2016; DEL DIEGO; GRACIA, 2012).

Desde la educación del personal de salud hasta la humanización de los servicios, el acompañamiento a los pacientes, su uso como terapias o como mecanismo para mejorar las condiciones laborales, las artes y las humanidades han tenido un papel importante en el contexto hospitalario (BLASCO-FONTECILLA, 2014; BORSAY, 2003; MASEL; SCHUR; WATZKE, 2012; STOKES, 1980; SÁNCHEZCAMUS, 2011) En algunos países europeos se han establecido programas estatales arte-humanidades-salud bajo la identificación de

\footnotetext{
"la necesidad de hacer del hospital un lugar más humano, abierto a la ciudad, se reconoce como una prioridad del sector médico y hospitalario. Esta necesidad se traduce en nuevas políticas que mejoren la acogida y el acompañamiento de las personas hospitalizadas y de sus familias, y que aseguren al personal sanitario un marco profesional más agradable. La cultura puede jugar un papel esencial en esta evolución. Sin intención de objetivos terapéuticos, esta participa en la mejora de la evolución de las personas y favorece la relación entre el hospital y su entorno" (DEL DIEGO; GRACIA, 2012, p. 215).
}

Adicionalmente las artes y las humanidades han sido identificados como herramientas en diversas áreas y formas: potencializadoras en la formación del personal de salud, para el acompañamiento en la enfermedad y el padecimiento, para la promoción de la salud de todos los actores involucrados en los escenarios clínicos, y como herramientas de investigación. Aunque las artes, las humanidades y la salud han estado vinculados desde el inicio de estas, la investigación de los efectos de estas prácticas solo se ha logrado consolidar, aunque en pequeña escala durante 
el último siglo. En 2019 la OMS- Región Europea publicó su informe sobre la evidencia del rol de las artes en la salud (FANCOURT; FINN, 2019) y presentando a partir del análisis de 900 publicaciones académicas las potencialidades del arte tanto en la salud física como mental. Hace énfasis en dos subtemas: la prevención y promoción de la salud, y el manejo y tratamiento de patologías. El informe previo señala cómo ha aumentado la investigación sobre los efectos de las artes en la salud y el bienestar desde inicio del presente siglo, pero a pesar de esto, no se cuenta con su posicionamento (FANCOURT, 2017).

En Colombia su inserción es reciente y poco se conoce o reporta sobre qué o cómo se hace. Este proyecto de investigación cualitativa denominado "Caracterización de las artes y humanidades en la formación de personal y prestación de servicios de salud", tuvo como objetivo identificar y describir las múltiples formas de aplicación de las artes y las humanidades en contextos hospitalarios y de formación profesional, realizadas en un Hospital Universitario y dos facultades de formación de profesionales de salud de la respectiva universidad en la ciudad de Bogotá-Colombia, para el periodo 2008 hasta 2018, así como las potencialidades y dificultades que este trabajo implica.

\section{Metodología}

El proyecto fue elaborado por tres coinvestigadoras, mujeres, de distintas disciplinas, vinculadas al campo de la salud y las humanidades tanto personal como académicamente. Todas tenían experiencia previa en investigación cualitativa. El proyecto fue financiado por la Convocatoria de proyectos interdisciplinares de la Pontificia Universidad Javeriana, y sometido y aprobado por el Comité de investigación y ética de la Facultad de Medicina y del Hospital Universitario San Ignacio, de la ciudad de Bogotá-Colombia.

El objetivo del proyecto se estableció al identificar en la propia institución universitaria, dentro de la cual las coinvestigadoras llevaban al momento del proyecto más de 15 años de trabajo contínuo, una serie de prácticas en donde se intersecaban las artes, las humanidades y la búsqueda o el mantenimiento de la salud, pero un vacío y desconocimiento sobre las mismas, tanto al interior de la propia institución como a nivel nacional. Para poder describir y comprender el lugar de estas prácticas dentro de la formación de profesionales de salud, se desarrolló un proyecto de carácter cualitativo, basado en entrevistas semiestructuradas a personas que, dentro de la Universidad o 
en el Hospital universitario, desarrollaran prácticas de aplicación o uso de las artes en la formación de estudiantes o en la atención a pacientes. Se contactaron a través del método de bola de nieve, y a través de dos convocatorias en redes institucionales y correos electrónicos distribuidos en las Facultades de Medicina y Enfermería, y al interior del Hospital Universitario, solicitando que profesores, estudiantes y directivos auto reportaran o identificaran experiencias o prácticas de terceros. A partir de este proceso se identificaron 30 personas susceptibles de ser entrevistadas, de las cuales 22 aceptaron la invitación. Con los 8 restantes no se puedo realizar las entrevistas principalmente por escases de tiempo. En un caso no se obtuvo respuesta alguna. Con estas 22 se realizaron entrevistas semiestructuradas y observación participante en algunas de las experiencias. En todos los casos se solicitó consentimiento informado individual y la autorización de los jefes de las respectivas unidades o facultades. Las entrevistas fueron realizadas por las coinvestigadoras, en parejas, siguiendo la guía de entrevista, y grabadas para posterior transcripción, en los espacios de trabajo de los entrevistados, o en espacios que el entrevistado se sintiera más cómodo. En todo caso la entrevista se hizo garantizando confidencialidad y sin la presencia de terceros. Se incluyeron estudiantes y profesores de las Facultades de Medicina y Enfermería, empleados del Hospital Universitario, estudiantes y profesores de otras facultades vinculados con estrategias que dan cuenta de esta intersección. Las entrevistas fueron realizadas a lo largo del año 2019, tenían una duración promedio de una hora y veinte minutos, y fueron transcritas a medida que fueron realizadas, por parte de 3 estudiantes de pregrado, supervisadas por las coinvestigadoras. Se garantizó el mantenimiento de la anonimidad acorde a lo que los entrevistados indicaron. En conjunto con las notas de campo y la revisión de literatura, el material fue analizado de manera manual por cada investigadora y triangulado con el equipo de trabajo en reuniones periódicas. El material fue organizado en tres categorías descriptivas previas identificadas en la revisión del estado del arte (COFFEY; ATKINSON, 2003) y presentadas aquí: artes y humanidades en la formación de personal de salud, el trabajo y acompañamiento a pacientes, y las artes y humanidades como objeto y herramienta de investigación, además de identificar su sentido y alcance desde la perspectiva de los propios actores. Las estrategias y prácticas identificadas son presentadas organizadas bajo esta lógica. Se incluyó una cuarta categoría, como categoría emergente que recoge las prácticas o experiencias que emergen en las intersecciones de las previamente establecidas. Los resultados fueron presentados a las distintas personas involucradas, 
buscando visibilizar los hallazgos y potenciar la creación de redes y trabajo entre distintas unidades. Adicionalmente se entregó un informe pormenorizado a las distintas unidades que servirá de línea de base para futuras evaluaciones de impacto o seguimiento, y sobre el cual pedimos retroalimentación en una sesión de grupo con los participantes.

\section{Resultados}

Los resultados aquí presentados reflejan la relación entre una institución universitaria y su correspondiente Hospital Universitario en donde buena parte de la formación de personal médico y de enfermería tiene lugar. Así como a la forma emergente como la relación entre arte, humanidades y salud ha tomado forma en el contexto de una universidad privada y confesional en Colombia. Y si bien las experiencia y prácticas identificadas no necesariamente acontecen todas dentro en el hospital, si hay un eje central en esta relación que permite su emergencia y en algunos casos sostenimiento en el tiempo.

\section{Las artes y humanidades en la formación profesional del personal de salud}

Los programas de pregrado incluidos en este estudio han pasado por dos reformas curriculares. Si embargo ambos coinciden en la inclusión de asignaturas de ética, epistemología de las respectivas disciplinas, constitución política y teología. Todas estas hacen parte del componente común cursado en todas las carreras de la Universidad. Si bien estas asignaturas existen los entrevistados reportan la sensación de no ser consideradas centrales ni por los estudiantes ni por muchos pares. Sin embargo, es un componente curricular transversal a todas las carreras de la Universidad y que se ha mantenido a lo largo de los últimos ańos como parte del enfoque integral en la formación. El programa de enfermería incluye asignaturas específicas como bioética (BADOUI, 2019) o antropología y salud. El programa de medicina si bien incluía estas asignaturas como independientes en la versión previa del programa, actualmente cuenta con esos contenidos incluidos en asignaturas integradoras que desarrollan múltiples dimensiones de la salud y la enfermedad a lo largo de cada semestre. En ambos casos los pregrados cuentan también con asignaturas de libre elección, ofertadas por diversas facultades y departamentos y en las cuales confluyen estudiantes de procesos de formación diversos. En relación con estas la principal queja de los estudiantes es la falta de tiempo para poder cursarlas 
o los cruces con aquellas que son obligatorias del programa a cursar. En el nivel de post grado en las especialidades médico-quirúrgicas, se hace mucho más énfasis en aspectos de formación en epistemología de las disciplinas, en especial en aquellas con fuerte base preventiva, o de intervención comunitaria como la geriatría o la psiquiatría. Sin embargo, el mayor peso de formación lo tienen las asignaturas en el marco del modelo anatomofisiológico de la enfermedad.

Otras prácticas asociadas a las artes y las humanidades identificadas fueron categorizadas como Prácticas pedagógicas individuales y currículo oculto (SUÁREZ OBANDO; DÍAZ AMADO, 2007), cuya característica fue el uso por parte de docentes de herramientas como la fotografía, el cine, el dibujo, la literatura, caricatura, videos cortos, entre otras; siempre con una intencionalidad no explicita, que uno de los participantes resume bien en un aparte de la entrevista: "Ha sido muy folclórico, digamos no hay una aproximación seria, sistemática, planeada, fundamentada a ese tema, no lo hay.." (Hombre, médico, profesor). En esta categoría se expresan aquellas prácticas o estrategias que aplican profesores particulares en su quehacer cotidiano, sin que necesariamente se reconozcan por la institucionalidad, o sin que necesariamente vean de manera favorable las artes y las humanidades. Una entrevistada nos dice: "Yo creo que en la facultad ha habido muchas cosas, pero todas como cosas aisladas, como entonces el seminario que teníamos sobre arte, entonces un profesor que hablaba de temas no sé del museo tal, o de la obra de fulano de tal, eran cosas como súper sueltas que había porque había personas que le gustaba o que le interesaba; sobre las raíces griegas o latinas de la terminología” (Entrevista a Mujer, médico, directivo). Los entrevistados reconocen además que aunque las recientes reformas curriculares de ambos programas de pregrado incluyen este tipo de asignaturas hay un currículo oculto que es el difícil de transformar: "Es como en una clase de bioética les dicen que hagan una cosa y al final hacen otra, como dice una cosa y en el hospital pasa otra, esa es una esquizofrenia y eso ocurre porque hay un currículo oculto que nunca se toca, el currículo oculto es el que esta intocado, (...) el currículo oculto nadie lo ha tocado.." (Entrevista a Hombre, médico, profesor).

\section{El trabajo con pacientes: artes y humanidades para sanar o acompañar dentro del Hospital}

En particular la corriente europea-inglesa ha explorado esta relación entre artes y humanidades, sus aplicaciones y usos actuales en el trabajo con los pacientes, 
su papel en la mejora de la relación personal de salud- paciente, o incluso el uso de las artes y las humanidades como herramienta para la generación de ingresos de las instituciones o de los pacientes, entendidas como formas de rehabilitación social y económica. En contextos de cuidado paliativo y cuidado oncológico, la presencia de la muerte y las limitaciones de las prácticas curativas biomédicas han encontrado en el arte y las humanidades un camino para mejorar las condiciones y calidad de vida de los pacientes, mejorar la relación médico paciente, bajar niveles de estrés del personal de salud e incorporar a familiares en el proceso (BLASCOFONTECILLA, 2014; BORSAY, 2003; MASEL; SCHUR; WATZKE, 2012; STOKES, 1980). En este sentido los resultados de este estudio identifican múltiples estrategias institucionalizadas dentro del Hospital. Al respecto un directivo nos dice en entrevista: “(...) toda institución se debe promover la creatividad y la innovación, la innovación en un hospital universitario suele ser muy cerrada a la innovación científica y nos quedamos en la innovación científica de las ciencias duras en una profesión que es humana. En ese sentido, siendo una ciencia humana debe aprender de otras disciplinas, como el aprendizaje desde las ciencias sociales(...) Pero si sabemos que es una ciencia humana y que la creatividad es fundamental en la innovación. La primera forma de abordar la creatividad es permitir o abordar espacios para que hagan manifestaciones de la creatividad artística de las personas: aquí estoy hablando de los trabajadores, de los estudiantes" (Entrevista a Hombre, Directivo, Hospital). Es así como al indagar con las distintas personas contactadas se identificó que el hospital cuenta con múltiples dependencias que vienen desarrollando en los últimos años actividades relacionadas con las artes y las humanidades. Dentro de las estrategias se incluyen: Acompańamientos a pacientes y familiares y empleados en momentos difíciles con apoyo de material creativo; protocolo de fin de vida y muerte que incluye actividades de escritura, creación y música en procesos de fin de vida; acompañamiento a familias en duelo por muerte desarrollado especialmente en la Unidad de Cuidado Paliativo; el programa educativo para cuidadores y el desarrollo de espacios de respiro para el personal de salud que incluyen actividades de trabajo corporal como yoga, respiración o danza; programas de divulgación de derechos y deberes haciendo uso de la música y el arte para alcanzar sus objetivos; así como el desarrollo y aplicación de la denominada 'humanización de la atención' que implica cambios sutiles en las relaciones interpersonales logrados a través de estrategias de comunicación, música y otras manifestaciones creativas, la aplicación de arte terapia 
y medidas no farmacológicas para la atención de cuidadores y pacientes de pacientes con trastornos neurocognitivos, y el acompañamiento espiritual desde la unidad de Pastoral de la Salud, en donde hacen uso de la lectura, la escucha, la música y la creación colectiva para establecer lazos y acompañamiento a los usuarios del hospital. Adicionalmente las expresiones artísticas permean las relaciones laborales, y se fomentan constantemente a través de concursos y espacios de encuentro.

En general las artes y las humanidades dentro del hospital tienen un lugar tanto para la salud de los trabajadores, como de los pacientes y cuidadores. Estas prácticas son bien valoradas y se reconoce su impacto positivo. Sin embargo, la mayor barrera presentada para su aplicación es la destinación de recursos tanto en infraestructura y económicos, como de tiempo y humanos. Si bien el personal de salud reconoce su potencial para la relación y acompañamiento con los pacientes insisten en "no tener tiempo", o no tener "el espíritu" que se requiere para su aplicación en contextos hospitalarios, señalando a unos pocos que parecen contar con aptitudes para hacerlo. Un trabajador del hospital nos dice: “...sin embargo hay otras dificultades que en efecto encontramos: primero, los mismos espacios. (haciendo referencia a los espacios reducidos dentro del hospital). Los mismos tiempos con los que nosotros contamos no son tiempos protegidos. A veces lo que trata de uno hacer es sacar el tiempo en medio de tantas ocupaciones, porque uno sabe que esa es la prioridad. Uno podría tener un asesor que dice 'yo lo puedo hacer', pero es que tengo tantos pacientes sin cubrir (...) Hay una guitarra para un guitarrista y a veces sacar los espacios pues no es fácil" (Entrevista a Hombre, teólogo, trabajador hospital).

\section{Artes y humanidades como método y objeto de investigación}

En este apartado se describen las experiencias en las que el arte es método u objeto de investigación, articulado a las actividades y funciones de la Universidad. Se encontraron grupos, líneas, proyectos y semilleros de investigación y otras actividades como seminarios. En la investigación cualitativa en salud, las humanidades y las artes se han empleado como herramientas de investigación, en particular en una corriente novedosa en el campo que es la investigación basada en las artes (DENZIN; LINCOLN, 2015), brindando nuevas comprensiones sobre el padecimiento, las formas de interpretación de la enfermedad, o la resignificación de estereotipos en contextos particulares. Su fundamento es profundamente interpretativo, entendiendo que las expresiones creativas permiten a las personas expresar niveles de la vida 
como es vivida, que lenguajes especializados o científicos limitan. Buena parte de la investigación realizada desde esta perspectiva se ha realizado desde las facultades de Ciencias Sociales, Filosofía e Instituto de Bioética. Sin embargo, mucho de este conocimiento es poco valorado desde la perspectiva biomédica. Un profesor, $\mathrm{PhD}$ en Filosofía nos cuenta durante la entrevista que, con años de trabajo en el tema del dolor, decidieron llevarlo a ambientes más clínicos y "entonces vamos y hablamos inicialmente con el Doctor X, del Instituto X de salud, y le dijimos: presentemos algo sobre esto. El Dr. dijo, eso no es científico, eso no es constatable, esto es básicamente carreta y no se pudo, no se pudo..." (Entrevista a Hombre, filósofo, profesor).

Por otro lado, existe una amplia producción de investigación dentro de la universidad que vincula expresiones artísticas con las neurociencias y neuropsicología. En el campo de la salud mental tanto el dibujo como la música son ampliamente utilizados (DEL DIEGO; GRACIA, 2012) bien como instrumento bien como objeto de investigación. De especial interés resulta el trabajo de Matallana y colaboradores (MATALLANA; MONTAÑEZ; SIERRA, 2012; MATALLANA; REYES; PALACIO; MORENO et al., 2011; MATALLANA ESLAVA, 2017) que explora la relación entre neurociencias y arte, así como investigaciones en intersección con la bioética y la antropologia (CALLEJAS DE LUCA, 2020; GUZMÁN, 2014). Finalmente en el periodo en cuestión estaban activos dos semilleros, que son espacios de formación de jóvenes investigadores de pregrado y postgrado, relacionados con las artes y las humanidades: el Semillero de donación de órganos y el semillero de artes, salud y humanidades, ambos con base en la Facultad de Medicina, en los que las artes y las humanidades se exploran como herramientas de intervención en salud en el primero, o como herramientas reflexivas y de investigación participativa en el segundo.

\section{El arte y la salud en espacios de intersección}

Estas son experiencias que surgen como resultado de la sinergia y trabajo conjunto entre la Universidad y el Hospital o entre diversas unidades de la Universidad, y no necesariamente como resultado interno de procesos dentro de las respectivas Facultades de Medicina o Enfermería. El trabajo de estudiantes, profesores y administrativos voluntarios es el que permite la existencia de varias de estas iniciativas y muchas, de hecho, surgen como propuestas individuales que se van consolidando con el tiempo. Se identificaron los escritos realizados por pacientes 
que reflexionan sobre su proceso de salud y enfermedad y que posteriormente son incorporados a algunas prácticas pedagógicas como la experiencia de vivir con cáncer o vivir con enfermedades huérfanas (DÁVILA BERMUDEZ, 2017; TAMAYO FERNANDEZ, 2019) otras surgen como iniciativas de estudiantes voluntarios que identifican en el sufrimiento del otro una opción para trabajar y realizar trabajo voluntario al interior del hospital, pero cuya iniciativa se concreta al lograr identificar recursos y capacidades de distintas unidades al interior de la Universidad y el Hospital que les permiten crear condiciones de posibilidad para su constitución y sostenimiento(LASERNA ESTRADA; BARAHONA CORREA; FIGUEROA GUTIÉRREZ; ZARATE VELASCO et al., 2016). Otras experiencias que resaltan por su impacto interinstitucional son el grupo Angie Baquiro, con su línea de trabajo de Clown intrahospitalario, que surge como grupo estudiantil de la Faculta de Medicina y progresivamente va articulando estudiantes de otras carreras, logrando el reconocimiento y apoyo de la Universidad. También Recital en el Hospital, como experiencia que lleva música a espacios poco convencionales dentro del hospital y vincula profesores, estudiantes y becarios, así como distintas unidades tanto del Hospital como de la Universidad y de la Rectoría. El Grupo de Apoyo Humanitario y el Coro Consentido que se basa en participación voluntaria de profesores o estudiantes para la transformación de espacios de atención en salud, pero que reúnen personas de diversas facultades y formaciones. Otros espacios como el Cine foro Inclusionarte: Dis-Capacidad ¿De quién?, se planteó como espacio de reflexión colectiva para la universidad, desde la iniciativa de una profesora de enfermería, para la reflexión y la discusión sobre la inclusión y la discapacidad dentro de la universidad, con el apoyo del Centro de Gestión cultural, una unidad asociada a la rectoría.

\section{El sentido}

Las humanidades interpelan y dan sentido a la existencia humana y al ejercicio profesional en sociedad. En una entrevista realizada a estudiantes de medicina del grupo Clown, señalan que las prácticas creativas dentro del hospital empoderan a los pacientes al permitirles decidir sobre asuntos de su vida en un espacio en donde pierden mucha autonomía y capacidad de decisión (en especial en unidades pediátricas). Un médico nos señala la potencia que tiene la interrelación de las artes y las humanidades desde una perspectiva emancipadora, de autoconocimiento, reflexión y crítica. Para 
los participantes se constituyen entonces dos polos: las artes y humanidades como recreativas y las artes y humanidades como potencialmente emancipadoras.

Adicionalmente una tercera postura cuestiona la medicina en sí, clasificándola como un arte con componente científico. Un médico especialista nos dice "Digamos que cuando uno empieza a aproximarse a la medicina, uno entra con una serie de prejuicios propios pues digamos de una persona de 18 ańos(...) pero a medida que se empieza a dar cuenta de que el objeto de la medicina es el ser humano , obligatoriamente en un escenario de interacción humana como es la relación médico paciente, empieza uno a descubrir componentes de arte del ejercicio médico que cada vez más lo obligan a uno a enfrentar la medicina no como una ciencia pura si no como con un arte con componente científico". (Entrevista a Hombre, médico, profesor) En este posicionamiento no existe una relación de dos campos o disciplinas distintos, sino que son constituyentes del ejercicio médico. No se concibe diferenciación entre una y otra.

\section{Discusión}

Se encontró que en su mayoría estas iniciativas subsisten por el empeño de personas que creen en ellas, que deben enfrentar la sobrevaloración de los aspectos anatomofisiológicos sobre los de la atención integral. Esta realidad se concreta en que muchas iniciativas que son realizadas por artistas cuenten con escaso tiempo y recurso humano y queden vinculadas al voluntariado y "tiempo libre".

En términos del componente académico, las estrategias o prácticas pedagógicas basadas en las artes o las humanidades son escasas, según lo reportan los participantes y la dificultad que supuso encontrar este tipo de experiencias. Según los entrevistados, desafortunadamente lo académico ha "diluido" las actividades culturales, artísticas o creativas institucionales pasándolas a un segundo plano. El cambio de currículo y la disminución del tiempo de clases presenciales han puesto en segundo lugar algunas de las estrategias pedagógicas, en donde estaba más presente el arte. Algunos participantes consideran como barreras predominantes para la vinculación del arte y las humanidades con las profesiones de la salud a la mentalidad tanto de estudiantes como de profesores a quienes se les dificulta comprender y valorar esta relación, pero que también está mediada por lo que el sistema impone. Respecto de esto último Aguilar (AGUILAR-FLEITAS, 2014) señala como la relación médico paciente hoy esta mediada por la tecnología y la burocracia y han institucionalizado esta relación 
generando una crisis que le ha impuesto a la medicina la necesidad de repensarse y recobrar áreas de interés como la bioética, la formación médica continua, la seguridad del paciente y las humanidades medicas entre otras. Las personas entrevistadas se debaten entre estas dos fuerzas: la hipertecnologización, la burocracia, la escasez de tiempo y la necesidad de conectarse y resignificar su labor diaria.

Percibimos en estas iniciativas una potencia que aplica a lo que llamamos ganagana, pues como experiencias atravesadas por encuentros intencionados de generar bienestar, o influir en el proceso formativo; quienes participan se transforman con la experiencia misma, sea porque son receptores de una propuesta o porque la ejecutan. En varias de las estrategias exploradas aparece una búsqueda existencial que encuentra sentido y lugar en ella, como la necesidad de trascender en la formación de personal de salud y descentrar el acercamiento al conocimiento sólo a través de herramientas de carácter positivista, formal y de medición; por ejemplo con el uso de casos concretos de personas que logran potencia artística después de un daño cerebral, se enseña a través de lo que una profesional nos relata cómo la potencia de "la paradoja del daño". O por medio del cine o la literatura se logra generar empatía y movilizar emoción, para enseñar que cuando se atiende a un paciente, no se evalúa la enfermedad sino el ser humano en su integridad y especialmente en su capacidad de aprender del sufrimiento. Hay un sentido de responsabilidad sobre la relación con el otro y como recibe lo que se hace; por ejemplo, como con el dibujo se potencia la participación y educación en salud en las poblaciones.

Los resultados -para las instituciones caracterizadas- son concordantes con lo publicado a nivel internacional (BARBER; MORENO-LEGUIZAMON, 2017). Aunque en Colombia -y en particular en estas instituciones- aún tienen un lugar subalterno, parecen posicionarse progresivamente al ser legitimadas desde los servicios y atención hospitalaria, como Cuidados Paliativos, Pediatría y cuidados a pacientes con enfermedades crónicas.

En su mayoría se sostienen a partir de iniciativas individuales y voluntariado, mostrando una progresiva institucionalización en los últimos 10 años con la aparición de programas financiados por ambas instituciones. Esto puede ser interpretado como un proceso de legitimación, pero que debe cuidarse de ser asociado exclusivamente al entretenimiento, perdiendo su sentido social y emancipador.

Si entendemos que el hospital universitario como lugar de formación y atención médico en todos los niveles de complejidad (ORTIZ MARTÍNEZ, 2016), este se 
convierte en un escenario donde las funciones de la universidad como formación, investigación, extensión y servicio se pueden concretar. En particular las estrategias desarrolladas al interior del hospital que involucran estudiantes y profesores producen cambios importantes en la percepción y relación que tienen los estudiantes con los pacientes, logrando salirse de un modelo exclusivamente anatomofisiológico y dando paso a una aproximación integral y basada en necesidades reales de pacientes, cuidadores y demás personal de salud. Es posible que estemos frente a una transformación paradigmática de los servicios de salud de una visión centrada en la patología, la enfermedad y las opciones quirúrgicas, a una formación centrada en los pacientes, sus contextos, y en la reconceptualización de "la salud" como integral, vinculante, transdisciplinar.

\section{Conclusiones}

En la formación de personal de salud tienen un papel central en términos bioéticos y de praxis clínica, pero muchas veces es interpretado como marginal, en la que se privilegian los saberes anatomo clínicos frente a la formación humanista, que requiere manejo del tiempo, la creatividad y el desarrollo integral de la persona, asuntos todos que pueden ir en contra de lógicas de formación y educación basadas en la competencia y la productividad.

Se encontró que las iniciativas que surgen como prácticas pedagógicas o investigativas logran tener un nivel de institucionalidad mayor, en tanto están vinculadas a labores que forman parte del trabajo y formas de evaluación de quienes las ejecutan.

El hospital universitario parece un escenario privilegiado, y poco explorado, para el desarrollo de la intersección, siempre y cuando se mantenga el espíritu critico y emancipatorio de las artes y las humanidades, además del recreativo y estético.

Se requiere mayor investigación para dar cuenta del valor de estas prácticas no solo para quienes las realizan sino para quienes las reciben y cómo se potencian sentidos de existencia y bienestar colectivos, además de continuar interpelando las maneras y estructuras de formación y atención en un campo tan sensible como el de la salud humana. También se requiere seguimento a recientes cambios introducidos en el programa, por fuera del período de estúdio, y cuyos efectos deberán evaluarse a mediano y largo plazo. 


\section{Agradecimientos}

Este artículo es producto del proyecto de investigación denominado "Caracterización de las artes y humanidades en la formación de personal y prestación de servicios de salud” financiado por la Pontificia Universidad Javeriana-Vicerrectoría de Investigación VRI-06-INTERDISC2018.

\section{Referências}

AGUILAR-FLEITAS, B. Humanidades Médicas. Su vigencia para la práctica clínica. Rev Urug Cardiol, 29, p. 69-172, 2014.

BADOUI, N. La formación en bioética en el pregrado de medicina: estudio de caso y propuesta para la Facultad de Medicina de la Pontificia Universidad Javeriana. O ¿Por qué y cómo formamos mejores ciudadanos en la facultad de medicina? Orientador: RUEDA BARRERA, E. 2019. (Maestría) - Maestría en Bioética, Pontificia Universidad Javeriana, Bogotá.

BARBER, S.; MORENO-LEGUIZAMON, C. J. Can narrative medicine education contribute to the delivery of compassionate care? A review of the literature. Medical Humanities, 2017.

BLASCO-FONTECILLA, H. Humanities in Medicine: Medicalization, wish-fulfilling medicine, and disease mongering: Toward a brave new world? Medicalización, medicina del deseo e invención de enfermedades: ¿hacia un mundo feliz? (Spanish; Castilian), 214, p. 104-107, 3/1/March 2014 2014. Personal Report.

BLEASE, C. In defence of utility: the medical humanities and medical education. BMJ Journals Medical Humanities, 2016-06-01 2016.

BORSAY, A. Medicine and Art. BMJ Journal Medical Humanities Online First, 2003-12-01 2003.

CALLEJAS DE LUCA, E. Representaciones sociales de un equipo médico de geriatría sobre el proceso de morir y su relación con la toma de decisiones frente a pacientes en final de vida.: Trabajo de grado pregrado Antropología y psicología, Pontificia Universidad Javeriana: 1-87 p. 2020.

COFFEY, A.; ATKINSON, P. Encontrar el sentido a los datos cualitativos. Estrategias complementarias de investigación. Medellin: Editorial Universidad de Antioquia., 2003.

DEL DIEGO, E.; GRACIA, A. CDAN. Arte Contemporáneo en el hospital. 2012. CDAN; salud mental; hospital; taller de artista; Huesca. 1988-2408|escape\}.

DENZIN, N.; LINCOLN, Y. Investigación con base en las artes. In: Manual de investigación Cualitativa. Barcelona: Gedisa Editorial, 2015. v. IV, p. 113 -139.

DÁVILA BERMUDEZ, M. C. En bus a Santa Marta. Colombia: Cain Press 2017.

FANCOURT, D. Arts in Health, Designing and researching interventions. Oxford, 2017. 
FANCOURT, D.; FINN, S. What is the evidence on the role of the arts in improving health and well-being? A scoping review. 2019.

GUZMÁN, C. El fracaso terapéutico": Un estudio Antropológico de la práctica de Cuidado Intensivo en el Hospital Universitario San Ignacio: tecnología, saberes y valores. 2014. (Pregrado) Departamento de Antropología, Pontificia Universidad Javeriana, Bogotá.

LASERNA ESTRADA, A.; BARAHONA CORREA, J.; FIGUEROA GUTIÉRREZ, L.; ZARATE VELASCO, A. et al. Biblioteca para pacientes del Hospital Universitario San Ignacio: ejemplo de liderazgo estudiantil. . Universitas Médica, 57, n. 1, p. 132-133., 2016.

MASEL, E. K.; SCHUR, S.; WATZKE, H. H. Humanities: Art, Language, and Spirituality in Health Care: Life is Uncertain. Death is Certain. Buddhism and Palliative Care. Journal of Pain and Symptom Management, 44, p. 307-312, 8/1/August 2012 2012. Article.

MATALLANA, D.; MONTAÑEZ, P.; SIERRA, N. Las demencias y la neuropsicología del dibujo. Bogotá: CEJA, 2012.

MATALLANA, D.; REYES, P.; PALACIO, K.; MORENO, T. et al. Demencia y creatividad: emergencia de una actividad pictórica en un paciente con afasia primaria progresiva. Revista Colombiana de Psiquiatría, 40, n. 4, p. 807-817, 2011/12/01 2011.

MATALLANA ESLAVA, D. L. Arte, cerebro y patología: Liberación de competencias artísticas. In: IV Congreso Nacional e Internacional de Geriatría y Gerontologia, 2017, Disponível em: http:// scienti.colciencias.gov.co:8081/cvlac/visualizador/generarCurriculoCv.do?cod_rh=0000055000.

ORTIZ MARTÍNEZ, J. G. Hospitales universitarios en Colombia: desde Flexner hasta los centros académicos de salud. Repertorio de Medicina y cirugía, 25, n. 1, p. 50-58, 2016.

PINTO, Z.; JORGE, R. Esa Curiosa Relación entre la Medicina y la Literatura. Horizonte Médico, 13, n. Octubre-diciembre, 2013.

STOKES, J. Grief and the Performing Arts: A Brief Experiment in Humanizing Medical Education. Journal of Medical Education, 55, n. 3, p. 215, 03/01/ 1980.

SUÁREZ OBANDO, F.; DÍAZ AMADO, E. La formación ética de los estudiantes de medicina: la brecha entre el currículo formal y el currículo oculto. Acta bioeth, v.13, n. 1, p. 107-113, Disponível em: https://scielo.conicyt.cl/scielo.php?script=sci_arttext\&pid=S1726569X2007000100011\&lng=es. http://dx.doi.org/10.4067/S1726-569X2007000100011.

SÁNCHEZ-CAMUS, R. The art of dying: Aesthetics and palliative care. Journal of Applied Arts \& Health, 2, n. 2, p. 155, 05// 2011.

TAMAYO FERNANDEZ, M. L. Trece historias huérfanas. Colombia: Fundación derecho a la desventaja 2019. 


\section{Resumo}

Artes e humanidades na formação de pessoal de saúde e na atenção integral em um hospital universitário

O objetivo deste artigo é identificar e descrever as múltiplas formas de aplicação das artes e humanidades em contextos hospitalares e de formação profissional nas Faculdades de Medicina e Enfermagem e em um Hospital Universitário da cidade de Bogotá-Colômbia no período de 2008 -2018. A caracterização foi feita a partir de métodos qualitativos, interpretativos, com aplicação de entrevistas semiestruturadas aos atores identificados por bola de neve e autorrelato. A observação participante também foi feita em alguns locais e práticas. Foram investigados os significados e usos das artes e humanidades em relação à saúde e à educação em saúde. Os diversos atores atribuem significados a essas práticas, relacionando-as a uma maior receptividade no processo ensino-aprendizagem, a possibilidade de gerar um sentido de transcendência sobre o que é feito, respondendo a questóes existenciais, proporcionando bem-estar às pessoas em condiçóes de hospitalização e em menor. medida para gerar bemestar próprio ou dos profissionais de saúde. Seu lugar nos contextos explorados ainda é percebido como subalterno, e eles são sustentados principalmente por iniciativas individuais e voluntariado. Deve-se insistir em sua legitimidade para avançar em seu sentido social e emancipatório.

> Palavras-chave: Humanidades, artes, saúde, educação, medicina, voluntários 


\section{Abstract}

\section{Arts and humanities in the training of health personnel and in comprehensive care in a university hospital}

The objective of this article is present the multiple forms of application of the arts and humanities in hospital contexts and in professional training in the Faculties of Medicine and Nursing and a University Hospital of the city of Bogotá-Colombia in the period 2008 -2018. The characterization was made from qualitative, interpretive methods, with application of semistructured interviews to actors identified by snowball and self-report. Participant observation was also made in some places and practices. The meanings and uses of the arts and humanities in relation to health and health education were investigated. The various actors assign meanings to these practices, relating them to a greater receptivity in the teaching-learning process, the possibility of generating a sense of transcendence about what is done, answering existential questions, providing well-being to people in hospitalization conditions and in less measure to generate own well-being or that of health professionals. Their place in the explored contexts is still perceived as subaltern, and they are mostly sustained by individual initiatives and volunteerism. Its legitimacy must be insisted upon in order to advance in its social and emancipatory sense.

Keywords: Humanities, arts, health, Education, medical, volunteers. 